\title{
Severe Acute Respiratory Syndrome Coronavirus 2 could exploit human transcription factors involved in Interferon-mediated response
}

Ighli di Bari

Universita degli Studi di Bari Aldo Moro

Rossana Franzin

Universita degli Studi di Bari Aldo Moro

Angela Picerno

Universita degli Studi di Bari Aldo Moro

Alessandra Stasi

Universita degli Studi di Bari Aldo Moro

Maria Teresa Cimmarusti

Universita degli Studi di Bari Aldo Moro

Mariagiovanna Di Chiano

Universita degli Studi di Bari Aldo Moro

Claudia Curci

Universita degli Studi di Bari Aldo Moro

Paola Pontrelli

Universita degli Studi di Bari Aldo Moro

Maria Chironna

Universita degli Studi di Bari Aldo Moro

\section{Giuseppe Castellano}

Universita degli Studi di Foggia Facolta di Medicina e Chirurgia

\section{Anna Gallone}

Universita degli Studi di Bari Aldo Moro

Carlo Sabbà

Universita degli Studi di Bari Aldo Moro

Loreto Gesualdo

Universita degli Studi di Bari Aldo Moro

Fabio Sallustio ( $\square$ fabio.sallustio@uniba.it)

University of Bari Aldo Moro https://orcid.org/0000-0002-5132-6532

\section{Research}

Keywords: SARS-CoV-2, COVID-19, ISGF3, transcriptional factors, interferon regulatory factors. 
Posted Date: July 23rd, 2020

DOI: https://doi.org/10.21203/rs.3.rs-43644/v1

License: (a) (i) This work is licensed under a Creative Commons Attribution 4.0 International License. Read Full License 


\section{Abstract}

Background The novel SARS-CoV-2 causing the pandemic acute respiratory disease COVID-19 is considered a worldwide emergency since no vaccines or effective antiviral are still available. As virus are dependent on the host transcriptional factors (TFs) to express the viral genes, efforts are required to understand the molecular interplay between virus and host response.

Methods By bioinformatic analysis, we investigated human TFs involved in viral transcription. In particular, we analyzed the key role of the TF induced by Interferon regulatory factors (IRFs) exploiting the way of antiviral response related to interferon antiviral cytokines released during an infection. We compared SARSCoV-2 consensus sequence from Italian patients with sequences in Coronaviridae ViPR database, to identify elements and TF binding sites that were present uniquely in the Italian viral sequence.

Results Several TFs were induced by IRFs, others were induced by IFN-stimulated gene factor 3 (ISGF3) and by un-phosphorylated ISGF3 that was found to promote the transcription of several viral open reading frame. These data shed light on SARS-CoV2 dependence from the host transcription machinery associated to interferon-related antiviral response. We found binding sites for $11 \mathrm{TF}$ present only in sequence of virus infecting humans and among these IRF8, IRF4, GLIS2, POU4F2 that were expressed in response to the viral infection and induced by interferon. POU4F2 binds in ORF1ab promoter, IRF8, and IRF4 both bind within the ORF1ab gene.

Conclusions Our data suggest that SARS-CoV-2 can exploit the interferon-related host response, inducing the expression of genes by host TF involved in the regulation of transcription by RNA polymerase Il, thus facilitating its own replication cycle.

These data strengthen our knowledge about the transcription and replicative activity of the virus and could pave the way for new targets drug design.

\section{Background}

Coronaviruses (CoV) are a large family of respiratory viruses that can cause mild to moderate diseases, from the common cold to respiratory syndromes such as MERS (Middle East respiratory syndrome) and SARS (Severe acute respiratory syndrome). Coronaviruses are common in many animal species (such as camels and bats) but in some cases, though rarely, they can evolve and infect humans and then spread to the population $[1,2]$.

Human coronaviruses known to date, common all over the world, are seven, some identified several years ago (the first in the mid-sixties) and some identified in the new millennium[3].

In 2019 a new strain of coronavirus that has never been identified before in humans, SARS-CoV-2, has been recognized. It is an RNA virus coated with a capsid and a peri-capsid crossed by glycoprotein structures that give it the typical 'crown' appearance, with a size of $100-160 \mathrm{~nm}$ in diameter [4]. It is part of the large coronavirus family and is genetically located within the Betacoronavirus genus, with a distinct clade in the $B$ lineage of the sub-genus Sarbecovirus as well as two non-human Sars-like strains [5]. In humans, the SARS- 
CoV-2 virus is capable of causing the disease called COVID-19, a severe acute respiratory syndrome leading to an high mortality rate and to several complications, including acute kidney injury in about $25 \%$ of patients [6]. The appearance of new pathogenic viruses for humans, previously circulating only in the animal world, is a widely known phenomenon (called spillover or jump of species) and it is thought that it may also be the basis of the origin of the new coronavirus SARS-CoV- 2.

At the moment, the scientific community is trying to identify the animal species from which the virus was transmitted to humans, even if most data suggest that the spillover originated by bats [1]. The SARS-CoV- 2 genome is constituted by a single strand of large-sized positive RNA (+ssRNA), about $30 \mathrm{~kb}$, that gives rise to 7 viral proteins and is associated with protein $\mathrm{N}$, which increases its stability(Figure 1A).

From the first Open Reading Frame (ORF) 1a/b start the viral proteins and about two-third of genome length, encodes 16 non-structural proteins (nsp1-16). Also a -1 frameshift is present between ORF1a and ORF1b and it is important for the processing of two polypeptides called pp1a and pp1ab. Nevertheless, there are four structural proteins that are essential for virion assembly and infection of CoVs. S proteins make up the spike on the surface of virus particles and they have a fundamental role on the viral attachment to host receptor $[7,8]$, the M protein is constituted of three transmembrane domains and it shapes the virions, promotes membrane curvature, and binds to the nucleocapsid $[9,10]$, the E protein plays an important role in virus assembly and release, and it is strongly implicated in the pathogenesis [11], the N protein is formed by two domains: they can bind virus RNA genome via dissimilar mechanisms (Figure 1B). N protein can bind nsp3 protein to promote "tie" the genome to replicase-transcriptase complex (RTC), and package the encapsidated genome into virions [2,12], and also, $\mathrm{N}$ can be an antagonist of interferon [13]. The positive RNA viruses manage host protein synthesis in order to limit the translation of cellular mRNAs and favor the synthesis of viral proteins $[14,15]$. Coronavirus has a polycistronic genome organization and it has a unique transcription mechanism to generate a nested set of subgenomic (sg) mRNAs, involved in expression of ORF sited downstream of the replicase ORF $1 \mathrm{a}$ and $1 \mathrm{~b}$. These sg-mRNAs all include at their 5 ' end a common leader sequence which implies that sg-mRNAs are synthesized by the fusion of noncontiguous sequences (leader and the 5 ' end of each one is called "the body") $[16,17]$.

Viruses are dependent on the host transcriptional machinery to express the first viral genes and the viral promoters/enhancers should be activated by the same signalling events as innate-immune genes. Viral control elements might functionally mimic innate immune enhancers, taking advantage of the activated immune signalling transcription factors (TF) for promoting viral gene expression.

Since many years it has been established that RNA viruses frequently subvert cellular factors for replication and transcription of viral RNAs. Many of these factors are normal components of cellular RNA processing or translation machineries, which are sabotaged to play a regulatory role in the replication and transcription of viral RNA. Therefore, cellular factors are active in the replication and transcription of viral RNAs. This has been experimentally proven by multiple experiments, for example the removal of the cellular factors invariably resulted in the loss of RNA-dependent RNA polymerase (RdRP) activity or template specificity. Viral RdRPs and cellular factors cooperate to form transcription or replication complexes on the viral RNA[18]. 
Here we investigated the human TF that can bind the SARS-CoV-2 sequence and found that several TF can be induced by the interferon antiviral response. This led to hypothesize that the virus may use this pathway to modulate the cellular RNA polymerase.

\section{Methods}

Selection of coronavirus sequences for comparison to 2019-nCoV

All full-length sequences from SARS-CoV Italian patients were retrieved from ViPR (https://www.viprbrc.org/brc/home.spg?decorator=corona) and from GISAID (https://www.gisaid.org/) databases on 30 march 2020. Sequences considered had the following ID:|EPI_ISL_412974; EPI_ISL_413489; EPI_ISL_417419; |EPI_ISL_417418; |EPI_ISL_417423; |EPI_ISL_417421; |EPI_ISL_412973;|EPI_ISL_417447. Sequences were aligned using the MUSCLE algorithm in ViPR. The consensus sequence of Italian virus was determined from the final alignments using the Sequence Variation Analysis tool in ViPR. Coronaviridae ViPR database was used to identify similar sequences using the BLAST algorithm to compute the pairwise identity between Italian consensus sequence and its comparison target.

\section{Identification of TF binding the SARS-CoV-2 sequence}

To identify TF binding the SARS-CoV-2 sequence we used the CiiiDER scanning [19] that utilizes an implementation of the MATCH algorithm [20] to predict potential TF binding sites in regions of interest. A deficit value of 0.15 , which is the difference between the MATCH score of a TF binding site and the maximum possible score, which is 1 , was used. For the scanning, position frequency matrices (PFMs) from the curated open-access JASPAR database $[21,22]$ were used. In order to predict whether a sequence contains a site for a TF, CiiiDER compared the virus sequence to the PFM and generated a score of similarity. If the sequence matches the PFM perfectly then the deficit value is 0 . To search for TF binding sites, sequences are split into overlapping five-base regions, which are compared with the core PFM. If the similarity score between a fivebase sequence and the core PFM meets a defined threshold, then the sequence window is increased to the full length of the TF binding sites and the similarity score with the full PFM is calculated. For the scanning we used the positive strand for ORF1 $a$ and $b$ and the negative strand for the remaining genes and ORF.An enrichment analysis, comparing the distribution of TF binding sites predicted in a set of regulatory regions to the distribution in a set of background sequences, has been utilised to more accurately identify TFBSs that are statistically over- or under-represented [23]. Over- and under-represented TFs were determined by comparing the numbers of sequences with predicted TF binding sites to the number of those without, using a Fisher's exact test; As background sequences we used binding sequences of TF induced by ISGF3 and by un-phosphorylated ISGF3 (U-ISGF3) [24].

\section{Results}

The human SARS-CoV-2 has $88 \%$ similarity with a Bat SARS-like coronavirus

The sequences of SARS-CoV-2 from 8 different Italian patients were multiple aligned and compared to identify a consensus sequence for the Italian viral strain. Then the consensus sequence was compared with 
all sequences included in the Coronaviridae ViPR database to identify similar sequences. The databases included 198 Species, 6938 genomes and 6642 strains. We found that our consensus sequence had a similarity at $88 \%$ with a Bat SARS-like coronavirus isolated in China, Igb|AVP78030 (Score $=1.995 \mathrm{e}+04$ bits, Supplementary Figure 1).

\section{Human TF potentially involved in SARS-CoV-2 transcription}

By using the Italian consensus sequence, we assessed the possible human TF able to bind the viral RNA. We considered the JASPAR vertebrate database. This model represents the majority of human TFs. A total of 659 TF belonging to Jaspar database and binding to sequences of SARS-CoV-2 was found (Supplementary Table 1). Then we selected TF that bind the viral sequence near the starting site of each of the viral gene or ORF considering a range of \pm 1000 bases from the starting sites (Supplementary Table 2 ). We found that the binding sites for TF were equally distributed between the promoter regions of each transcribed sequence except for the ORF1 $1 \mathrm{ab}$ and ORF10, in which TF binding sites were under-represented (Figure1C). Only 4.2\% of the TF can bind the ORF1ab and $6.7 \%$ can bind the ORF10.

\section{TF induced by IFN}

Several genes are activated by viral infection following induction of interferon release by host cells. Therefore, we performed an enrichment analysis to identify TF induced by IFN and whose binding sites were overrepresented in the Italian SARS-CoV-2 sequence. In particular, we investigated TF involved in the activation of IFN-stimulated gene factor 3 (ISGF3), which drives the expression of more than 100 IFNß-stimulated genes [25]. We subdivided genes and TF induced by ISGF3 and those induced by un-phosphorylated ISGF3 (UISGF3), that maintains the expression of a subset of the initially induced interferon stimulated genes (ISGs), whose protein products lead to extended resistance to virus infection and DNA damage. We found that SARS-CoV-2 contains 18 binding sites for 11 ISGF3-induced TF located nearby the transcription starting sites of virus genes (Table 1 and Figure 2). Most of ISGF3-induced TFs bound promoters of $S$ protein and of the ORF3a whereas no TF bound the ORF1ab (Figure 2). Moreover, the same TF, Six1, could bind the promoter regions of genes codifying for structural proteins $E$ and $M$ and of ORF 6, 7, 8, and 9 . However, 36 different binding sites for 19 U-ISGF3-induced TF were present (Table 2 and Figure 3). Among these, seven different UISGF3-induced TF bound the ORF3a, four TF bound the promoter of the gene codifying for the S protein and three TF bound the ORF1ab. SOX21 and HOXA9 could bind three different promoter regions of ORF3 and genes $\mathrm{E}$ and $\mathrm{M}$, whereas FOXK1 and Hmx1 could bind five different promoter regions of ORF6, ORF7, ORF8, ORF9, and $\mathrm{N}$ gene. Among U-ISGF3-induced TF is included again SIX1 that bind promoter regions of $4 \mathrm{ORF}$ and 2 genes of the virus (Figure 3 ).

Binding sites of TF involved in regulation of transcription by RNA polymerase II are present exclusively in SARS-CoV-2 genome isolated from humans

We then compared TF binding sites present in the SARS-CoV-2 sequence isolated from Italian patients (Supplementary Table 1) and those present in the sequence of Bat SARS-like coronavirus (Supplementary table 3). Interestingly, we found that 12 TF binding sites were present exclusively in the genomic region of 
SARS-CoV-2 isolated from humans and not in the virus isolated from the bat (Table 3). Among these factors, some were involved in the interferon response, as Interferon Regulatory Factor 4 (IRF4), Interferon Regulatory Factor 8 (IRF8), and POU Class 4 Homeobox 2 (POU4F2). Moreover, we performed a network analysis and found that ten of twelve TF formed a significant network $(p=3.53 e-05$, Figure 4$)$. In addition, the functional enrichment analysis showed that most significant biological processes in which these genes are involved were constituted by the regulation of transcription by RNA polymerase II (false discovery rate $=2.34 \mathrm{e}-10$ 3.56e-09; Table 4).

\section{Discussion}

Coronavirus RNA synthesis is performed by a multienzymatic replicase complex together with cellular factors and the process requires the specific recognition of RNA cis-acting signals located in the viral genome. Cellular proteins are involved in coronavirus RNA synthesis together with the p100 transcriptional co-activator protein [26]. A strong interaction between the host cell and the virus replication and transcription processes exists in virus of the Coronaviridae family [27]. In absence of a specific mechanisms for the control of the cell and its replicative machinery for the synthesis of viral products, the viral genome could overcome cellular genes by competition. To overwhelm the cell and focus the transcription on the viral genome, viruses exploits several mechanisms. They can selectively inhibit host cell transcription or eliminate host mRNA and they also can encode their own set of transcriptional activators which facilitate the assembly of the host cell transcription complex at the site of RNA initiation on the viral genome [28]. A virus must counteract intrinsic cellular and innate-immune responses to successfully complete the replication cycle. The viral promoters/enhancers can be activated by the same signalling events as innate-immune genes.

Coronaviruses gives rise to mRNAs that are structurally similar to those of their eukaryotic hosts, and this allows them to parasitize the host machinery to translate the viral mRNA. Specific factors that interfere with host translation or transcription or other factors that enhance viral-specific synthesis are responsible for the increase of virus-encoded molecules [29].

Recently, it has also been experimentally demonstrated how extensively the host is involved in the SARS-CoV2 replication / transcription activity. Indeed, more than 500 host proteins constituting the replication/transcription complex (RTC) microenvironment have been identified [30] [31]. Among these biochemically validated cellular factors, 8 TF that we identified in our analysis were present (STAT1, STAT3, TFEB, NFIX, NFIC, Stat5a, NR3C1,Zfx).

Viral IE control elements might functionally mimic innate immune enhancers, gaining advantage from TF activated by immune signalling to induce viral IE gene expression [32]. Here we found that SARS-CoV-2 genome is enriched in binding sites for TF activated by Interferon- $\beta$. Interferons (IFNs), such as IFN- $a$, IFN- $\beta$, and IFN-y, are important antiviral cytokines released during an infection. These cytokines are activated once the immune cells are faced with an infection. Recent studies have showed that some viruses exploit cisacting elements and/or TFs related to IFN-induced antiviral activities with the aim of enhancing their replicative processes. In the case of these specific viruses, the IFN response process also facilitates viral replication and gene expression. For example, the genome of human immunodeficiency virus (HIV), a sSRNA 
virus, contains motifs that are similar to the consensus binding sites for TFs belonging to the host immune genes [33,34].

The HIV promoter has an important role in viral gene expression and several host factors have been demonstrated to contribute to the virus activity and replication. Indeed, HIV core promoter is the central key point for all signals that repress or active viral transcription since it pirates the host cell RNA polymerase II (Pol II) machinery to initiate viral transcription [35]. Coronavirus-host interactions play a crucial role in viral pathogenesis and infection. The coronavirus nonstructural proteins (nsp), in collaboration with recruited host cell proteins, constitute a membrane-associated replication and transcription complexes. SARS-CoV nsp1 was the first CoV nsp1 that was shown to block the expression of reporter gene under the control of IFN- $\beta$ promoter [36]. From our analysis, a key role of some transcriptional factors such as the family of interferon regulatory factors (IRFs) has emerged. They are a group of transcription factors that are related to the regulation of gene expression and the immune response. In the IRF family, formed by ten members, nine (IRF1-IRF9) have been recognised in mammals, and specifically in humans [37].

In particular we found two set of TF: one is induced by the TF ISGF3 (IRF9 and tyrosine-phosphorylated STATs 1 and 2) that drives the first rapid cellular response to the viral infection; the other is induced by the related factor un-phosphorylated ISGF3 (U-ISGF3) that drives the second prolonged response. U-ISGF3 is composed by IFNb-induced IRF9 and STATs 1 and 2 without tyrosine phosphorylation. The U-ISGF3-induced anti-viral genes that show prolonged expression are driven by distinct IFN stimulated response elements (ISRES). These TF activated by the SARS-CoV-2, support our hypothesis that, mimicking an innate-immune enhancer, they can be directly utilised in a timed manner to guarantee viral gene expression at IE times [32]. Interestingly, our data are also supported by the recent discovery that SARS-CoV-2 Receptor ACE2 is an interferon-stimulated gene and that SARS-CoV-2 could exploit species-specific interferon-driven upregulation of ACE2 to enhance infection [38].

One of the IFN-dependent TF that can bind several domains of the SARS-CoV-2 genome is SIX1. It plays a critical role in embryogenesis, is not expressed in normal adult tissue, but is expressed in many malignancies, including cervical [39] and HIV-associated lung cancer [40]. However, Hypoxia and HIF-1a can increase SIX1 expression, which reciprocally stimulates HIF-1a expression under both normoxic and hypoxic conditions, thereby creating a positive feedback regulatory loop [41]. Therefore, it is plausible that the hypoxic conditions in SARS-CoV patients contribute to the viral activity by the activation of SIX1 that, in turn, can bind viral ORFs and genes.

Another factor that can bind SARS-CoV-2, HOXA9, has an important role for the $\mathrm{C}$ glycoprotein synthesis in the Varicella Zoster Virus. Indeed, PBX / HOXA9 heterodimers bind the promoter region of this viral glycoprotein and HOXA9 homodimer binds the promoter of another viral protein which may upregulate $\mathrm{C}$ glycoprotein synthesis [42].

Moreover, we found a set of TF whose binding sites are present in SARS-CoV- 2 but not in the Bat SARS-like coronavirus despite they have a similarity of $88 \%$. 
As shown in our analysis, Interferon Regulatory Factor 4 and 8 (IRF 4 and 8) could be involved with a positive virus interaction. Many RNA viruses are able to induce host translational shut-off, inhibiting the synthesis of peptides and proteins that are necessary for cell response to infection. Many of the RNA viruses, including Caliciviridae, Coronaviridae, Picornaviridae, Oorthomyxoviridae, Reoviridae, and many others, have strategies to induce host translational shut-off and thus prevent the infected cells to synthesize new peptides and proteins, including those IFN-stimulated IRFs and STATs. IRF2 and IRF4 have been implicated in the suppression of type I IFN signaling. [43,44]. IRF-4 and ZNF148 TF are involved in Epstein - Barr virus (EBV) infections. IRF-4 is involved in maintaining the growth phenotypes of EBV-transformed cells in vitro [45]. However, ZNF148 is activated by an early lytic protein encoded by the EBV BMRF1 gene [46]. Therefore SARS-CoV2 could be able to control host translational machinery by IRF-4 and suppress IFN signaling in infected cells.

Interferon regulatory factor 8 (IRF8) is a TF of the IRF family that is induced by interferon in an innate response against infections. This TF is activated by IFN-g in macrophages and stimulates genes essential for a host response. The cellular factor IRF8 facilitates EpsteinBar virus lytic replication by promoting caspase expression and their activation upon lytic induction, in contrast with the most of the IRFs activities that contribute to anti-viral immunity and block the infection or lytic reactivation of herpesviruses [47-50].

Another important TF is the POU Class 4 Homeobox 1 (POU4F2, Brn-3b). It binds in ORF1ab promoter. POU4F2 expression is involved in the activation of immediate-early promoters of herpes simplex virus (HSV) and varicella zoster virus (VZV) leading to virus gene transcription and replication [51]. It is involved also in the transcription of human papilloma virus [52]. The retinoid $X$ receptor a ( $R X R a)$ may increase host susceptibility to viral infections by suppressing type I interferon [53,54]. From our network analysis RXRA results linked to the receptor for retinoic acid RARG and to THRB. The overexpression of THRB (TRB1) and its regulatory effects has been reported to occur during Herpes virus infection (HSV) [55-57]. TR 1 is a member of the thyroid hormone receptors (TRs) family and it is most abundant in the liver, kidney and the inner ear [58]. In undifferentiated cells, the overexpression of TR $\beta 1$ was associated to a remarkably viral replication [57]. Therefore, it would be more interesting to study in the future the interplay between TR 1 and SARS-COV2 replication in order to provide important insights into the development of treatments for COVID-19 syndrome.

Additional studies are needed to further validate these findings.

Finally, our functional enrichment analysis showed that several TF, implicated in interferon-mediated response and whose binding sites were specifically found in the sequence of SARS-COV-2 infecting humans, are involved in the regulation of transcription by RNA polymerase II, suggesting a process similar to that of HIV regarding the recruiting of RNA Polimerase II and TFs machinery of the host to initiate viral transcription [59-61].

\section{Conclusions}

The presence of a strong interaction between the host cell and transcription processes exists in virus of the Coronaviridae family. This prompted us to investigate and understand the molecular interplay between virus 
and host response.

Our data suggest that SARS-CoV-2 can exploit the interferon-related host response, inducing the expression of genes by host TF involved in the regulation of transcription by RNA polymerase II, thus facilitating its own replication cycle.

These data strengthen our knowledge about the transcription and replicative activity of the virus, help to understand the mechanisms of interaction between SARS-CoV-2 and host, act as a starting point for further in-depth studies and could pave the way for new targets drug design.

\section{Abbreviations}

Coronaviruses (CoV)

Epstein - Barr virus (EBV)

Herpes Simplex Virus (HSV)

Human Immunodeficiency Virus (HIV)

IFN stimulated response elements (ISREs)

IFN-stimulated gene factor 3 (ISGF3)

Interferon Regulatory Factors (IRFs)

Interferon Regulatory Factor 4 (IRF4)

Interferon Regulatory Factor 8 (IRF8)

Interferon Stimulated Genes (ISGs)

Interferons (IFNs)

Middle East Respiratory Syndrome (MERS)

non-structural proteins (nsp)

Open Reading Frame (ORF)

Position Frequency Matrices (PFMs)

POU Class 4 Homeobox 2 (POU4F2)

POU Class 4 Homeobox 2 (POU4F2)

Replicase-Transcriptase Complex (RTC) 
Retinoid X Receptor a (RXRa)

RNA polymerase II (Pol II)

RNA-dependent RNA polymerase (RdRP)

Severe acute respiratory syndrome (SARS)

Single Strand positive RNA (+ssRNA)

subgenomic (sg)

THRB (TRß1)

Thyroid hormone Receptors (TRs)

Transcription Factors (TF)

Un-phosphorylated ISGF3 (U-ISGF3)

Varicella Zoster Virus (VZV)

\section{Declarations}

Ethics approval and consent to participate

Not applicable

Consent for publication

Not applicable

Availability of data and materials

Not applicable

\section{Competing interests}

The authors declare that the research was conducted in the absence of any commercial or financial relationship that could be construed as a potential conflict of interest.

\section{Funding}

Not applicable

\section{Authors' contributions}

F. Sallustio and I. di Bari planned the research, coordinated the study, designed and performed bioinformatics analysis, analyzed the respective data and drafted the manuscript; A. Stasi, A. Picerno, M.T. Cimmarusti, C. 
Curci, M. Di Chiano, and R. Franzin participated in the design of the study and assisted in manuscript preparation; P. Pontrelli, A. Gallone, G. Castellano, M. Chironna and C. Sabbà participated in the coordination of the study and assisted in manuscript preparation;C. Sabbà, F. Sallustio, and L. Gesualdo designed and supervised the research and drafted the manuscript; and all authors read and approved the final manuscript.

\section{Acknowledgements}

This work was supported by University of Bari "Aldo Moro" and the Italian Ministry of Health (AIM-181005 to A. Stasi).

\section{References}

1. Rabaan AA, Al-Ahmed SH, Haque S, Sah R, Tiwari R, Malik YS, et al. SARS-CoV-2, SARS-CoV, and MERSCoV: a comparative overview. Le Infez Med. 2020;2:174-84.

2. Fehr AR, Perlman S. Coronaviruses. An overview of their replication and pathogenesis. Coronaviruses Methods Protoc. 2015.

3. de Wilde AH, Snijder EJ, Kikkert M, van Hemert MJ. Host factors in coronavirus replication. Curr Top Microbiol Immunol. 2018.

4. Rezza G, Bella A, Riccardo F, Pezzotti P, D’Ancona F. OP. Coronavirus e nuovo coronavirus SARS-CoV-2. 2020.

5. Luk HKH, Li X, Fung J, Lau SKP, Woo PCY. Molecular epidemiology, evolution and phylogeny of SARS coronavirus. Infect. Genet. Evol. 2019.

6. 10.1186/s13054-020-02872-z

Fanelli V, Fiorentino M, Cantaluppi V, Gesualdo L, Stallone G, Ronco C, et al. Acute kidney injury in SARSCoV-2 infected patients. Crit Care [Internet]. BioMed Central; 2020 [cited 2020 May 7];24:155. Available from: https://ccforum.biomedcentral.com/articles/10.1186/s13054-020-02872-z.

7. Beniac DR, Andonov A, Grudeski E, Booth TF. Architecture of the SARS coronavirus prefusion spike. Nat Struct Mol Biol. 2006.

8. Delmas B, Laude H. Assembly of coronavirus spike protein into trimers and its role in epitope expression. J Virol. 1990.

9. Nal B, Chan C, Kien F, Siu L, Tse J, Chu K, et al. Differential maturation and subcellular localization of severe acute respiratory syndrome coronavirus surface proteins S, M and E. J Gen Virol. 2005.

10. Neuman BW, Kiss G, Kunding AH, Bhella D, Baksh MF, Connelly S, et al. A structural analysis of M protein in coronavirus assembly and morphology. J Struct Biol. 2011.

11. Nieto-Torres JL, DeDiego ML, Verdiá-Báguena C, Jimenez-Guardeño JM, Regla-Nava JA, FernandezDelgado R, et al. Severe Acute Respiratory Syndrome Coronavirus Envelope Protein Ion Channel Activity Promotes Virus Fitness and Pathogenesis. PLoS Pathog. 2014.

12. Hurst KR, Koetzner CA, Masters PS. Identification of In Vivo-Interacting Domains of the Murine Coronavirus Nucleocapsid Protein. J Virol. 2009. 
13. Cui L, Wang H, Ji Y, Yang J, Xu S, Huang X, et al. The Nucleocapsid Protein of Coronaviruses Acts as a Viral Suppressor of RNA Silencing in Mammalian Cells. J Virol. 2015.

14. Walsh D, Mohr I. Viral subversion of the host protein synthesis machinery. Nat. Rev. Microbiol. 2011.

15. Fung TS, Liao Y, Liu DX. Regulation of stress responses and translational control by coronavirus. Viruses. 2016.

16. Sawicki SG, Sawicki DL. Coronaviruses use discontinuous extension for synthesis of subgenome-length negative strands. Adv. Exp. Med. Biol. 1995.

17. Sola I, Almazán F, Zúñiga S, Enjuanes L. Continuous and Discontinuous RNA Synthesis in Coronaviruses. Annu Rev Virol. 2015.

18. Lai MM. Cellular factors in the transcription and replication of viral RNA genomes: a parallel to DNAdependent RNA transcription. Virology [Internet]. Virology; 1998 [cited 2020 Jun 23];244:1-12. Available from: http://www.ncbi.nlm.nih.gov/pubmed/9581772.

19. http://dx.plos.10.1371/journal.pone.0215495

Gearing LJ, Cumming HE, Chapman R, Finkel AM, Woodhouse IB, Luu K, et al CiiiDER: A tool for predicting and analysing transcription factor binding sites. Helmer-Citterich $\mathrm{M}$, editor. PLoS One [Internet]. Public Library of Science; 2019 [cited 2020 May 1];14:e0215495. Available from:

http://dx.plos.org/10.1371/journal.pone.0215495.

20. $10.1093 / \mathrm{nar} / \mathrm{gkg} 585$

Kel AE. MATCHTM: a tool for searching transcription factor binding sites in DNA sequences. Nucleic Acids Res [Internet]. 2003 [cited 2020 Apr 21];31:3576-9. Available from: https://academic.oup.com/nar/article-lookup/doi/10.1093/nar/gkg585.

21. 10.1093/nar/gkh012

Sandelin A, Alkema W, Engström P, Wasserman WW, Lenhard B. JASPAR: an open-access database for eukaryotic transcription factor binding profiles. Nucleic Acids Res [Internet]. 2004 [cited 2020 Apr 21];32:D91-4. Available from: https://academic.oup.com/nar/article-lookup/doi/10.1093/nar/gkh012.

22. Khan A, Fornes O, Stigliani A, Gheorghe M, Castro-Mondragon JA, van der Lee R, et al. JASPAR 2018 : update of the open-access database of transcription factor binding profiles and its web framework. Nucleic Acids Res [Internet]. 2018 [cited 2020 Apr 21];46:D260-6. Available from:

http://academic.oup.com/nar/article/46/D1/D260/4621338.

23. 10.3389/fgene.2016.00024/abstract

Boeva V. Analysis of Genomic Sequence Motifs for Deciphering Transcription Factor Binding and Transcriptional Regulation in Eukaryotic Cells. Front Genet [Internet]. 2016 [cited 2020 Apr 21];7:24. Available from: http://journal.frontiersin.org/Article/10.3389/fgene.2016.00024/abstract.

24. 10.1038/emboj.2013.203

Cheon H, Holvey-Bates EG, Schoggins JW, Forster S, Hertzog P, Imanaka N, et al. IFNß-dependent increases in STAT1, STAT2, and IRF9 mediate resistance to viruses and DNA damage. EMBO J [Internet]. 2013 [cited 2020 Apr 21];32:2751-63. Available from:

http://emboj.embopress.org/cgi/doi/10.1038/emboj.2013.203. 
25. Borden EC, Sen GC, Uze G, Silverman RH, Ransohoff RM, Foster GR, et al. Interferons at age 50: Past, current and future impact on biomedicine. Nat. Rev. Drug Discov. 2007.

26. Galán C, Sola I, Nogales A, Thomas B, Akoulitchev A, Enjuanes L, et al. Host cell proteins interacting with the $3^{\prime}$ end of TGEV coronavirus genome influence virus replication. Virology. 2009.

27. 10.1128/JVI.00321-13

Emmott E, Munday D, Bickerton E, Britton P, Rodgers MA, Whitehouse A, et al. The Cellular Interactome of the Coronavirus Infectious Bronchitis Virus Nucleocapsid Protein and Functional Implications for Virus Biology. J Virol [Internet]. 2013 [cited 2020 Apr 18];87:9486-500. Available from:

http://jvi.asm.org/cgi/doi/10.1128/JVI.00321-13.

28. Rohrmann G. Baculovirus Molecular Biology Baculovirus Molecular Biology Baculovirus Molecular Biology. Baculovirus Mol Biol. 2008.

29. Enjuanes L, Almazán F, Sola I, Zuñiga S. Biochemical aspects of coronavirus replication and virus-host interaction. Annu Rev Microbiol [Internet]. Annu Rev Microbiol; 2006 [cited 2020 Jun 23];60:211-30. Available from: http://www.ncbi.nlm.nih.gov/pubmed/16712436.

30. V'kovski P, Gerber M, Kelly J, Pfaender S, Ebert N, Braga Lagache S, et al. Determination of host proteins composing the microenvironment of coronavirus replicase complexes by proximity-labeling. Elife [Internet]. 2019 [cited 2020 Jun 23];8. Available from: https://elifesciences.org/articles/42037.

31. van Hemert MJ, van den Worm SHE, Knoops K, Mommaas AM, Gorbalenya AE, Snijder EJ. SARScoronavirus replication/transcription complexes are membrane-protected and need a host factor for activity in vitro. PLoS Pathog [Internet]. PLoS Pathog; 2008 [cited 2020 Jun 23];4:e1000054. Available from: http://www.ncbi.nlm.nih.gov/pubmed/18451981.

32. Kropp KA, Angulo A, Ghazal P. Viral Enhancer Mimicry of Host Innate-Immune Promoters. PLoS Pathog. 2014.

33. Mutthi P, Theerawatanasirikul S, Roytrakul S, Paemanee A, Lekcharoensuk C, Hansoongnern P, et al. Interferon gamma induces cellular protein alteration and increases replication of porcine circovirus type 2 in PK-15 cells. Arch Virol. 2018.

34. Shokouh Makvandi-Nejad. Human Immunodeficiency Virus (HIV).

35. Wilhelm E, Bell B. Selective recognition of viral promoters by host cell transcription complexes: Challenges and opportunities to control latency. Curr. Opin. Virol. 2013.

36. Narayanan K, Ramirez SI, Lokugamage KG, Makino S. Coronavirus nonstructural protein 1: Common and distinct functions in the regulation of host and viral gene expression. Virus Res [Internet]. Virus Res; 2015 [cited 2020 Jun 23];202:89-100. Available from: http://www.ncbi.nlm.nih.gov/pubmed/25432065.

37. Nam S, Lim JS. Essential role of interferon regulatory factor 4 (IRF4) in immune cell development. Arch. Pharm. Res. 2016.

38. Ziegler CGK, Allon SJ, Nyquist SK, Mbano IM, Miao VN, Tzouanas CN, et al. SARS-CoV-2 Receptor ACE2 Is an Interferon-Stimulated Gene in Human Airway Epithelial Cells and Is Detected in Specific Cell Subsets across Tissues. Cell [Internet]. Cell; 2020 [cited 2020 Jun 23];181:1016-1035.e19. Available from: http://www.ncbi.nlm.nih.gov/pubmed/32413319. 
39. Hosseinipour M, Wan F, Altomare D, Creek KE, Pirisi L. HPV16-transformed human keratinocytes depend on SIX1 expression for proliferation and HPV E6/E7 gene expression. Virology. Academic Press Inc.; 2019;537:20-30.

40. Zheng J, Wang L, Cheng Z, Pei Z, Zhang Z, Li Z, et al. Molecular Changes of Lung Malignancy in HIV Infection. Sci Rep. Nature Publishing Group; 2018;8.

41. Xu H, Zhang Y, Peña MM, Pirisi L, Creek KE. Six1 promotes colorectal cancer growth and metastasis by stimulating angiogenesis and recruiting tumor-associated macrophages. Carcinogenesis [Internet]. 2017 [cited 2020 Apr 28];38:281-92. Available from: http://www.ncbi.nlm.nih.gov/pubmed/28199476.

42. Storlie J, Jackson W, Hutchinson J, Grose C. Delayed biosynthesis of varicella-zoster virus glycoprotein C: upregulation by hexamethylene bisacetamide and retinoic acid treatment of infected cells. J Virol [Internet]. 2006 [cited 2020 Apr 28];80:9544-56. Available from: https://jvi.asm.org/content/80/19/9544.

43. Chiang H, Sen, Liu HM. The molecular basis of viral inhibition of IRF-and STAT-dependent immune responses. Front. Immunol. 2019.

44. Bentz GL, Liu R, Hahn AM, Shackelford J, Pagano JS. Epstein-Barr virus BRLF1 inhibits transcription of IRF3 and IRF7 and suppresses induction of interferon- $\beta$. Virology. 2010.

45. Xu D, Zhao L, Del Valle L, Miklossy J, Zhang L. Interferon Regulatory Factor 4 Is Involved in Epstein-Barr Virus-Mediated Transformation of Human B Lymphocytes. J Virol. 2008.

46. Holley-Guthrie EA, Seaman WT, Bhende P, Merchant JL, Kenney SC. The Epstein-Barr Virus Protein BMRF1 Activates Gastrin Transcription. J Virol [Internet]. 2005 [cited 2020 Apr 26];79:745-55. Available from: https://jvi.asm.org/content/79/2/745.

47. Lv DW, Zhang K, Li R. Interferon regulatory factor 8 regulates caspase-1 expression to facilitate EpsteinBarr virus reactivation in response to $B$ cell receptor stimulation and chemical induction. PLoS Pathog. 2018.

48. Twum DYF, Colligan SH, Hoffend NC, Katsuta E, Gomez EC, Hensen ML, et al. IFN regulatory factor-8 expression in macrophages governs an antimetastatic program. JCI Insight. 2019.

49. Zhang XJ, Jiang DS, Li H. The interferon regulatory factors as novel potential targets in the treatment of cardiovascular diseases. Br. J. Pharmacol. 2015.

50. Tamura T, Yanai H, Savitsky D, Taniguchi T. The IRF Family Transcription Factors in Immunity and Oncogenesis. Annu Rev Immunol. 2008.

51. Brownlees J, Gough G, Thomas S, Watts P, Cohen J, Coffin R, et al. Distinct responses of the herpes simplex virus and varicella zoster virus immediate early promoters to the cellular transcription factors Brn-3a and Brn-3b. Int J Biochem Cell Biol [Internet]. 1999 [cited 2020 Apr 26];31:451-61. Available from: https://linkinghub.elsevier.com/retrieve/pii/S1357272598001514.

52. Ndisang D, Faulkes DJ, Gascoyne D, Lee SA, Ripley BJ, Sindos M, et al. Differential regulation of different human papilloma virus variants by the POU family transcription factor Brn-3a. Oncogene [Internet]. Nature Publishing Group; 2006 [cited 2020 Jun 25];25:51-60. Available from: http://www.nature.com/articles/1209006.

53. Aguilar-Jimenez W, Zapata W, Rivero-Juárez A, Pineda JA, Laplana M, Taborda NA, et al. Genetic associations of the vitamin $\mathrm{D}$ and antiviral pathways with natural resistance to HIV- 1 infection are 
influenced by interpopulation variability. Infect Genet Evol [Internet]. 2019 [cited 2020 Apr 26];73:276-86. Available from: https://linkinghub.elsevier.com/retrieve/pii/S1567134819300929.

54. Ma F, Liu S-Y, Razani B, Arora N, Li B, Kagechika H, et al. Retinoid X receptor a attenuates host antiviral response by suppressing type I interferon. Nat Commun [Internet]. 2014 [cited 2020 Apr 26];5:5494. Available from: http://www.nature.com/articles/ncomms6494.

55. Figliozzi RW, Chen F, Balish M, Ajavon A, Hsia SV. Thyroid hormone-dependent epigenetic suppression of herpes simplex virus-1 gene expression and viral replication in differentiated neuroendocrine cells. $J$ Neurol Sci. 2014.

56. Bedadala GR, Pinnoji RC, Palem JR, Hsia SCV. Thyroid hormone controls the gene expression of HSV-1 LAT and ICPO in neuronal cells. Cell Res. 2010.

57. Chen F, Figliozzi RW, Bedadala G, Palem J, Hsia SV. Overexpression of thyroid hormone receptor $\beta 1$ altered thyroid hormone-mediated regulation of herpes simplex virus-1 replication in differentiated cells. J Neurovirol. 2016.

58. Anyetei-Anum CS, Roggero VR, Allison LA. Thyroid hormone receptor localization in target tissues. J. Endocrinol. 2018.

59. Ott M, Geyer M, Zhou Q. The control of HIV transcription: Keeping RNA polymerase II on track. Cell Host Microbe. 2011.

60. Roebuck KA, Saifuddin M. Regulation of HIV-1 transcription. Gene Expr. 1999.

61. Ne E, Palstra RJ, Mahmoudi T. Transcription. Insights From the HIV-1 Promoter. Int Rev Cell Mol Biol. 2018.

\section{Tables}

Table 1.

SARS-CoV-2 binding sites for ISGF3-induced Transcription Factors (TFs). 


\begin{tabular}{c|c|c|c|c|c|c|c|cc}
\hline Gene & Start & $\begin{array}{c}\text { Transcription } \\
\text { Factor Name }\end{array}$ & $\begin{array}{c}\text { Transcription } \\
\text { Factor ID }\end{array}$ & $\begin{array}{c}\text { Start } \\
\text { Position }\end{array}$ & $\begin{array}{c}\text { End } \\
\text { Position }\end{array}$ & $\begin{array}{c}\text { Core } \\
\text { Match } \\
\text { Score }\end{array}$ & $\begin{array}{c}\text { Matrix } \\
\text { Match } \\
\text { Score }\end{array}$ & Sequence \\
\hline S & 21563 & HOXD9 & MA0913.2 & 21578 & 21587 & 1 & 1 & GCAATAAAAC \\
\hline S & 21563 & HOXD13 & MA0909.2 & 21578 & 21588 & 1 & 0,993 & GGCAATAAAAC \\
\hline S & 21563 & CDX4 & MA1473.1 & 21578 & 21588 & 1 & 1 & GGCAATAAAAC \\
\hline ORF3a & 25393 & Alx1 & MA0854.1 & 24591 & 24607 & 1 & 0,98 & AGCTCTAATTAATTGTT \\
\hline ORF3a & 25393 & SOX21 & MA0866.1 & 26089 & 26103 & 1 & 0,91 & AACAATTTTATTGTA \\
\hline ORF3a & 25393 & TGIF2LY & MA1572.1 & 24799 & 24810 & 1 & 0,896 & TGACAAATGGCA \\
\hline E & 26245 & SIX1 & MA1118.1 & 27220 & 27230 & 1 & 0,995 & GTAACCTGAAA \\
\hline E & 26245 & SOX21 & MA0866.1 & 26089 & 26103 & 1 & 0,91 & AACAATTTTATTGTA \\
\hline M & 26523 & SIX1 & MA1118.1 & 27220 & 27230 & 1 & 0,995 & GTAACCTGAAA \\
\hline M & 26523 & SOX21 & MA0866.1 & 26089 & 26103 & 1 & 0,91 & AACAATTTTATTGTA \\
\hline ORF6 & 27202 & SIX1 & MA1118.1 & 27220 & 27230 & 1 & 0,995 & GTAACCTGAAA \\
\hline ORF7a & 27394 & SIX1 & MA1118.1 & 27220 & 27230 & 1 & 0,995 & GTAACCTGAAA \\
\hline ORF7b & 27756 & SIX1 & MA1118.1 & 27220 & 27230 & 1 & 0,995 & GTAACCTGAAA \\
\hline ORF8 & 27894 & SIX1 & MA1118.1 & 27220 & 27230 & 1 & 0,995 & GTAACCTGAAA \\
\hline N & 28274 & SHOX & MA0630.1 & 28490 & 28497 & 1 & 1 & TTAATTGG \\
\hline N & 28274 & JUN & MA0488.1 & 27290 & 27302 & 1 & 0,965 & TTTATGATGTAAT \\
\hline ORF10 & 29558 & Bach1::Mafk & MA0591.1 & 29511 & 29525 & 1 & 0,923 & GAGTTGAGTCAGCAC \\
\hline ORF10 & 29558 & MAFA & MA1521.1 & 29327 & 29341 & 1 & 0,923 & ATGCTTATTCAGCAA
\end{tabular}

Table 2.

SARS-CoV-2 binding sites for unphosphorylated ISGF3-induced Transcription Factors (TFs). 


\begin{tabular}{|c|c|c|c|c|c|c|c|c|}
\hline Gene & Start & $\begin{array}{l}\text { Transcription } \\
\text { Factor Name }\end{array}$ & $\begin{array}{l}\text { Transcription } \\
\text { Factor ID }\end{array}$ & $\begin{array}{c}\text { Start } \\
\text { Position }\end{array}$ & $\begin{array}{c}\text { End } \\
\text { Position }\end{array}$ & $\begin{array}{l}\text { Core } \\
\text { Match } \\
\text { Score }\end{array}$ & $\begin{array}{l}\text { Matrix } \\
\text { Match } \\
\text { Score }\end{array}$ & Sequence \\
\hline orf1ab & 266 & POU4F2 & MA0683.1 & 125 & 140 & 1 & 0,858 & CAGTATAATTAATAAC \\
\hline orf1ab & 266 & RFX2 & MA0600.2 & 659 & 674 & 0,89 & 0,855 & GGTGGCCATAGTTACG \\
\hline orf1ab & 266 & XBP1 & MA0844.1 & 385 & 398 & 1 & 0,95 & AGAGGCACGTCAAC \\
\hline $\mathrm{S}$ & 21563 & HOXD9 & MA0913.2 & 21578 & 21587 & 1 & 1 & GCAATAAAAC \\
\hline $\mathrm{S}$ & 21563 & ATOH1(var.2) & MA1467.1 & 22336 & 22345 & 1 & 0,957 & ACCAGCTGTC \\
\hline $\mathrm{S}$ & 21563 & HOXD13 & MA0909.2 & 21578 & 21588 & 1 & 0,993 & GGCAATAAAAC \\
\hline $\mathrm{S}$ & 21563 & Stat5a & MA1624.1 & 21178 & 21189 & 1 & 0,999 & ATTCCAAGAATG \\
\hline $\mathrm{S}$ & 21563 & CDX4 & MA1473.1 & 21578 & 21588 & 1 & 1 & GGCAATAAAAC \\
\hline ORF3a & 25393 & NEUROG2 & MA0669.1 & 24577 & 24586 & 1 & 0,957 & CACATATGTC \\
\hline ORF3a & 25393 & Six3 & MA0631.1 & 24491 & 24507 & 1 & 0,904 & GAAAGGATATCATTTAA \\
\hline ORF3a & 25393 & ZSCAN4 & MA1155.1 & 24647 & 24661 & 1 & 0,888 & TACACACTCTGACAT \\
\hline ORF3a & 25393 & NEUROG1 & MA0623.2 & 24577 & 24586 & 1 & 0,971 & CACATATGTC \\
\hline ORF3a & 25393 & Alx1 & MA0854.1 & 24591 & 24607 & 1 & 0,98 & AGCTCTAATTAATTGTT \\
\hline ORF3a & 25393 & SOX21 & MA0866.1 & 26089 & 26103 & 1 & 0,91 & AACAATTTTATTGTA \\
\hline ORF3a & 25393 & HOXA9 & MA0594.2 & 25848 & 25857 & 1 & 0,99 & GTCGTAACAA \\
\hline $\mathrm{E}$ & 26245 & SIX1 & MA1118.1 & 27220 & 27230 & 1 & 0,995 & GTAACCTGAAA \\
\hline $\mathrm{E}$ & 26245 & SOX21 & MA0866.1 & 26089 & 26103 & 1 & 0,91 & AACAATTTTATTGTA \\
\hline $\mathrm{E}$ & 26245 & HOXA9 & MA0594.2 & 25848 & 25857 & 1 & 0,99 & GTCGTAACAA \\
\hline M & 26523 & SIX1 & MA1118.1 & 27220 & 27230 & 1 & 0,995 & GTAACCTGAAA \\
\hline M & 26523 & SOX21 & MA0866.1 & 26089 & 26103 & 1 & 0,91 & AACAATTTTATTGTA \\
\hline M & 26523 & HOXA9 & MA0594.2 & 25848 & 25857 & 1 & 0,99 & GTCGTAACAA \\
\hline ORF6 & 27202 & SIX1 & MA1118.1 & 27220 & 27230 & 1 & 0,995 & GTAACCTGAAA \\
\hline ORF6 & 27202 & FOXK1 & MA0852.2 & 28136 & 28149 & 1 & 0,993 & AAGGTAAACAGGAA \\
\hline ORF6 & 27202 & Hmx1 & MA0896.1 & 28151 & 28167 & 1 & 0,955 & CCTGGCAATTAATTGTA \\
\hline ORF7a & 27394 & SIX1 & MA1118.1 & 27220 & 27230 & 1 & 0,995 & GTAACCTGAAA \\
\hline ORF7a & 27394 & FOXK1 & MA0852.2 & 28136 & 28149 & 1 & 0,993 & AAGGTAAACAGGAA \\
\hline ORF7a & 27394 & Hmx1 & MA0896.1 & 28151 & 28167 & 1 & 0,955 & CCTGGCAATTAATTGTA \\
\hline ORF7b & 27756 & SIX1 & MA1118.1 & 27220 & 27230 & 1 & 0,995 & GTAACCTGAAA \\
\hline ORF7b & 27756 & FOXK1 & MA0852.2 & 28136 & 28149 & 1 & 0,993 & AAGGTAAACAGGAA \\
\hline ORF7b & 27756 & Hmx1 & MA0896.1 & 28151 & 28167 & 1 & 0,955 & CCTGGCAATTAATTGTA \\
\hline ORF8 & 27894 & SIX1 & MA1118.1 & 27220 & 27230 & 1 & 0,995 & GTAACCTGAAA \\
\hline ORF8 & 27894 & FOXK1 & MA0852.2 & 28136 & 28149 & 1 & 0,993 & AAGGTAAACAGGAA \\
\hline ORF8 & 27894 & Hmx1 & MA0896.1 & 28151 & 28167 & 1 & 0,955 & CCTGGCAATTAATTGTA \\
\hline $\mathrm{N}$ & 28274 & FOXK1 & MA0852.2 & 28136 & 28149 & 1 & 0,993 & AAGGTAAACAGGAA \\
\hline $\mathrm{N}$ & 28274 & Hmx1 & MA0896.1 & 28151 & 28167 & 1 & 0,955 & CCTGGCAATTAATTGTA \\
\hline ORF10 & 29558 & MAFA & MA1521.1 & 29327 & 29341 & 1 & 0,923 & ATGCTTATTCAGCAA \\
\hline
\end{tabular}

\section{Table 3.}

scription Factor binding sites present exclusively in the genome of SARS-CoV-2 isolated from humans. 


\begin{tabular}{c|c|c|c|c|c|c|}
\hline $\begin{array}{c}\text { Transcription } \\
\text { Factor Name }\end{array}$ & $\begin{array}{c}\text { Transcription } \\
\text { Factor ID }\end{array}$ & $\begin{array}{c}\text { Start } \\
\text { Position }\end{array}$ & $\begin{array}{c}\text { End } \\
\text { Position }\end{array}$ & $\begin{array}{c}\text { Core } \\
\text { Match } \\
\text { Score }\end{array}$ & $\begin{array}{c}\text { Matrix } \\
\text { Match } \\
\text { Score }\end{array}$ & Sequence \\
\hline $\begin{array}{c}\text { THRB(var.2) } \\
\text { MA1575.1 }\end{array}$ & 5940 & 5958 & 1 & 0,858 & TTGTCCAACTTAGGGTCAA \\
\hline IRF4 & MA0736.1 & 21633 & 21646 & 1 & 0,85 & TACCCCCTGCATAC \\
\hline RXRA::VDR & MA0074.1 & 14280 & 14294 & 1 & 0,855 & CGGTCAAAGAGTTTT \\
\hline IRF8 & MA0652.1 & 4687 & 4700 & 1 & 0,894 & AAGAAACAGAAACT \\
\hline ZNF148 & MA1653.1 & 797 & 808 & 1 & 0,878 & TGCCCCTCCGTT \\
\hline THRB(var.3) & MA1576.1 & 8312 & 8330 & 1 & 0,853 & ATGACACCCCGTGACCTTG \\
\hline MYBL2 & MA0777.1 & 23192 & 23206 & 1 & 0,868 & GCCTGTTAAACCATT \\
\hline Rarg(var.2) & MA0860.1 & 8313 & 8329 & 1 & 0,865 & AAGGTCACGGGGTGTCA \\
\hline HES6 & MA1493.1 & 23494 & 23503 & 1 & 0,937 & TGCACGTGTT \\
\hline POU4F2 & MA0683.1 & 125 & 140 & 1 & 0,858 & CAGTATAATTAATAAC \\
\hline TP53 & MA0106.3 & 18281 & 18298 & 1 & 0,867 & TCCATGCACGTACATGTC
\end{tabular}

Table 4.

Functional enrichment analysis: most significant biological processes in which TF are involved.

\begin{tabular}{l|l|l|l|} 
GO-TERM & DESCRIPTION & COUNT IN GENE SET & FALSE DISCOVERY RATE \\
\hline
\end{tabular}

\begin{tabular}{|c|c|c|c|}
\hline$\underline{\mathrm{G}} \underline{\mathrm{O}: 0045944}$ & positive regulation of transcription by RNA polymerase II & 11 of 1104 & $2.34 \mathrm{e}-10$ \\
\hline$\underline{G} \underline{0: 0006366}$ & transcription by RNA polymerase II & 10 of 784 & $3.69 \mathrm{e}-10$ \\
\hline$\underline{G} \underline{0: 0000122}$ & negative regulation of transcription by RNA polymerase II & 10 of 809 & $3.69 \mathrm{e}-10$ \\
\hline$\overline{\mathrm{G}} \overline{\mathrm{O}: 0051254}$ & positive regulation of RNA metabolic process & 11 of 1596 & $1.85 \mathrm{e}-09$ \\
\hline$\underline{\mathrm{G}} \underline{\mathrm{O}: 0006357}$ & regulation of transcription by RNA polymerase II & 12 of 2633 & $3.56 \mathrm{e}-09$ \\
\hline$\underline{\mathrm{G}} \underline{\underline{O: 0006351}}$ & transcription, DNA-templated & 12 of 2569 & $3.56 \mathrm{e}-09$ \\
\hline$\underline{\mathrm{G}} \underline{\mathrm{O}: 0051253}$ & negative regulation of RNA metabolic process & 10 of 1303 & $5.48 e-09$ \\
\hline$\overline{\mathrm{G}} \underline{\underline{\mathrm{O}: 0016070}}$ & RNA metabolic process & 12 of 3430 & $3.02 \mathrm{e}-08$ \\
\hline$\overline{\mathrm{G}} \overline{\mathrm{O}: 0051252}$ & regulation of RNA metabolic process & 12 of 3890 & $1.05 e-07$ \\
\hline$\underline{\mathrm{G}} \underline{\mathrm{O}: 0043401}$ & steroid hormone mediated signaling pathway & 5 of 131 & $2.69 \mathrm{e}-07$ \\
\hline$\underline{\mathrm{G}} \underline{\mathrm{O}: 0006367}$ & transcription initiation from RNA polymerase II promoter & 5 of 162 & $6.16 \mathrm{e}-07$ \\
\hline$\underline{G} \underline{0: 0030522}$ & intracellular receptor signaling pathway & 5 of 173 & $7.65 e-07$ \\
\hline$\overline{\mathrm{G}} \overline{\mathrm{O}: 0048869}$ & cellular developmental process & 11 of 3533 & $1.13 e-06$ \\
\hline$\underline{\mathrm{G}} \underline{\mathrm{O}: 0071396}$ & cellular response to lipid & 6 of 486 & $3.00 \mathrm{e}-06$ \\
\hline$\underline{\mathrm{G}} \underline{\mathrm{O}: 0071310}$ & cellular response to organic substance & 9 of 2219 & $7.38 \mathrm{e}-06$ \\
\hline$\underline{\mathrm{G}} \underline{\underline{O: 0048523}}$ & negative regulation of cellular process & 11 of 4454 & $1.15 e-05$ \\
\hline$\overline{\mathrm{G}} \overline{\mathrm{O}: 0030154}$ & cell differentiation & 10 of 3457 & $1.89 \mathrm{e}-05$ \\
\hline
\end{tabular}

\section{Figures}




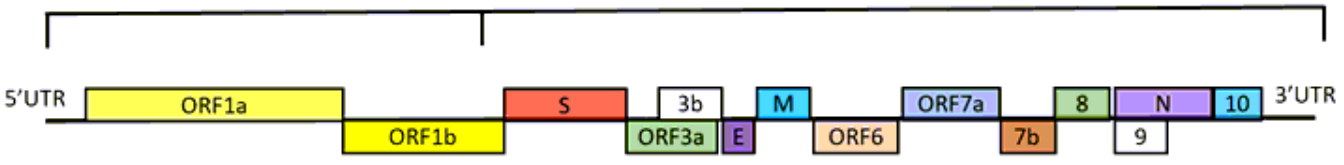

B

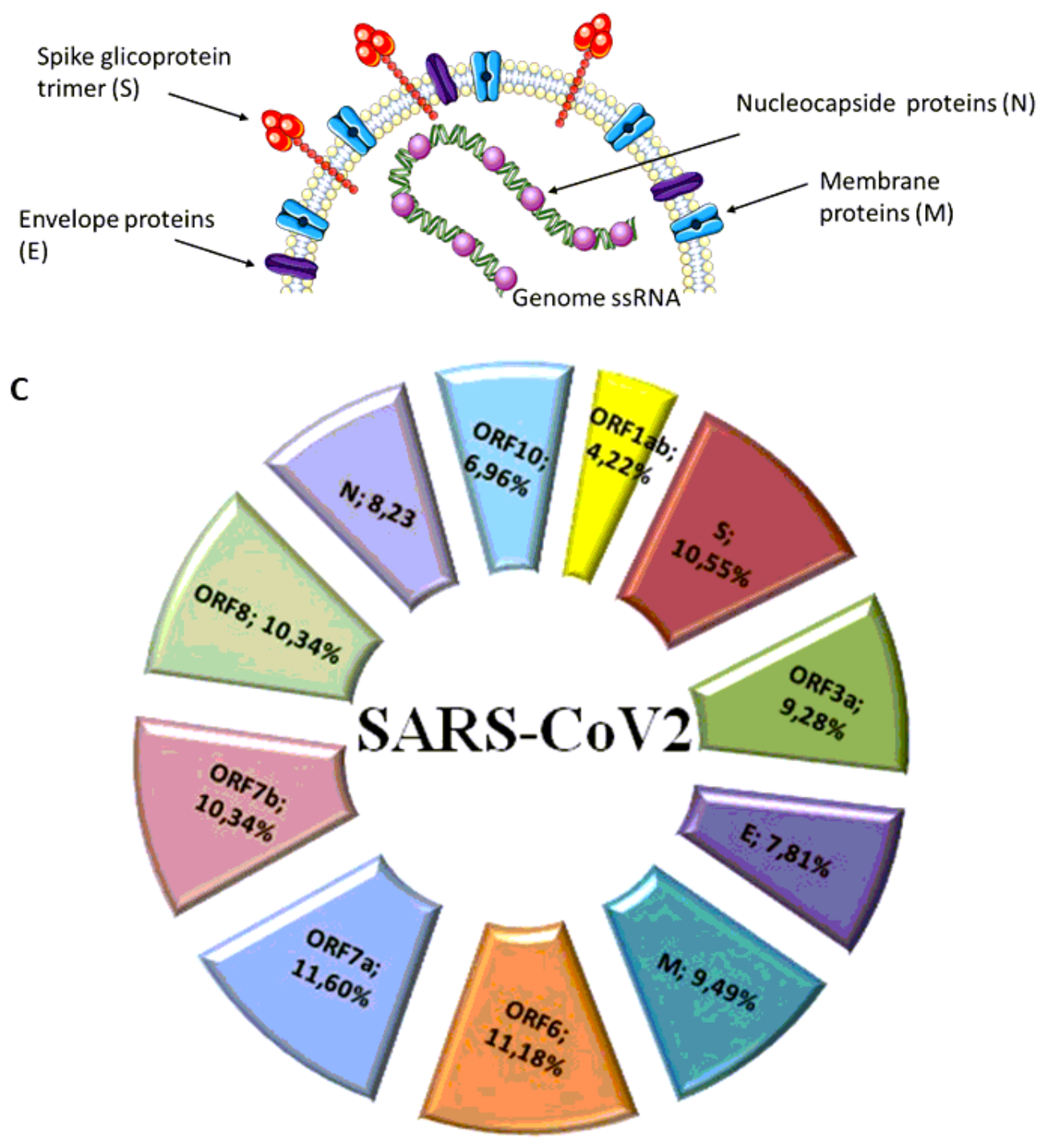

\section{Figure 1}

Schematic overview of genome, genes and proteins of SARS-CoV-2. (A) SARS-CoV-2 genome is formed a positive- sense, single- stranded RNA (ssRNA) genome of 27-32 kb in size. The 5'-terminal (translated from the first ORF1a and ORF1b) encodes two large polyproteins, pp1a and pp1ab, which are proteolytically cleaved into 16 non structural proteins (nsps), including papain-like protease (PLpro), $3 \mathrm{C}$ like protease (3CLpro), RNA-dependent RNA polymerase (RdRp). An additional 9-12 ORFs are encoded through the transcription of a nested set of subgenomic RNAs. The 3 ' terminus encodes structural proteins, including 
envelope glycoproteins spike (S), envelope (E), membrane (M) and nucleocapsid (N). (B) Coronavirus are enveloped and spherical particles of 100-160 nm in diameter. The envelope glycoprotein spike (S) forms a layer of glycoproteins trimer with the typical crown like appearence. Two additional transmembrane glycoproteins are incorporated in the virion: envelope (E) and membrane (M). Inside the viral envelope resides the helical nucleocapsid, which consists of the viral positive-sense RNA ((+)RNA) genome encapsidated by protein nucleocapsid (N). (C) Percentage distribution of transcription Factors (TFs) binding sites in the different genomic regions of the SARS-COV2. ORF1ab: Open Reading Frame 1ab, S: protein S, ORF3a: Open Reading Frame 3a, E: protein E, M: Protein M, ORF6: Open Reading Frame 6, ORF7a: Open Reading Frame 7a, ORF7b: Open Reading Frame 7b, ORF8: Open Reading Frame 8, N: protein N, ORF10: Open Reading Frame 10.

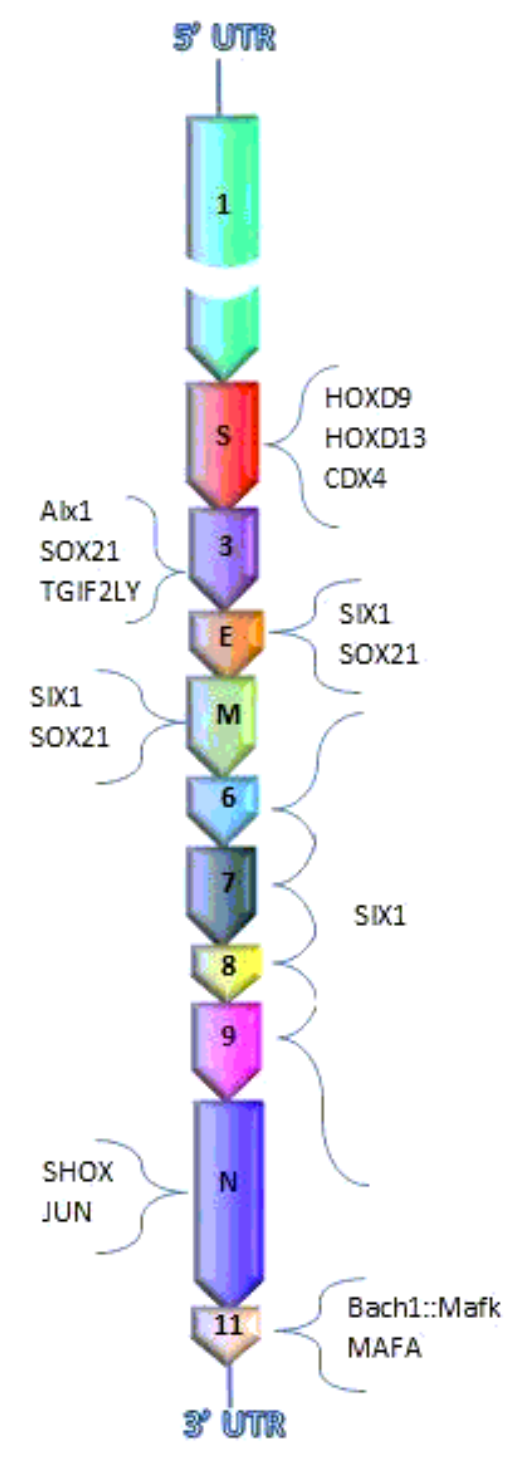

\begin{tabular}{|l|}
\hline 1- ORF1ab \\
3- ORF3a \\
6- ORF6 \\
7- ORF7a \\
8- ORF7b \\
9- ORF8 \\
11- ORF10 \\
JASPAR - ISGF3
\end{tabular}

\section{Figure 2}

ISGF3-induced Transcription Factors (TFs) whose binding sites are present in the different genomic regions of SARS-CoV-2. (1) ORF1ab: Open Reading Frame 1ab, S: protein S, (3) ORF3a: Open Reading Frame 3a, E: protein E, M: Protein M, (6) ORF6: Open Reading Frame 6, (7) ORF7a: Open Reading Frame 7a, (8) ORF7b: Open Reading Frame 7b, (9) ORF8: Open Reading Frame 8, N: protein N, (11) ORF10: Open Reading Frame 10. 


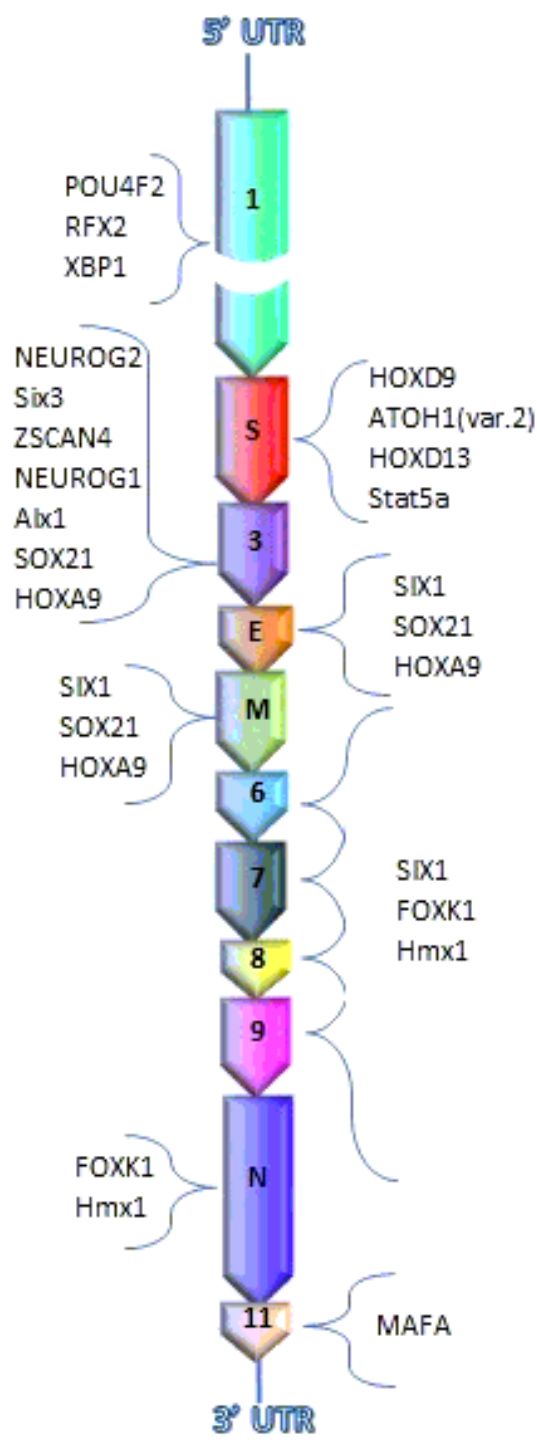
1. ORF1ab
3. ORF3a
6. ORF6
7. ORF7a
8- ORF7b
9. ORF8
11- ORF10

JASPAR - U-ISGF3

\section{Figure 3}

Unphosphorylated ISGF3 (U-ISGF3) induced Transcription Factors (TFs) whose binding sites are present in the different genomic regions of SARS-CoV-2. (1) ORF1ab: Open Reading Frame 1ab, S: protein S, (3) ORF3a: Open Reading Frame 3a, E: protein E, M: Protein M, (6) ORF6: Open Reading Frame 6, (7) ORF7a: Open Reading Frame 7a, (8) ORF7b: Open Reading Frame 7b, (9) ORF8: Open Reading Frame 8, N: protein N, (11) ORF10: Open Reading Frame 10. 


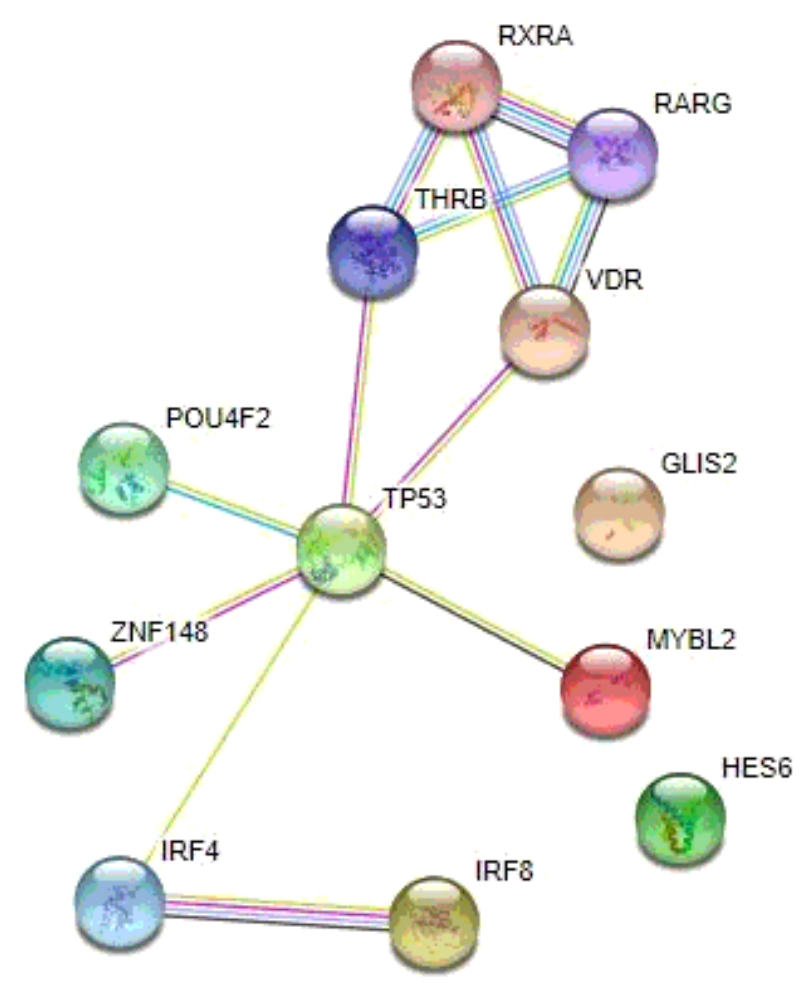

Figure 4

Network analysis of transcription factors binding exclusively the genomic region of SARS-CoV-2 isolated from humans. Ten of twelve TF formed a significant network $(p=3.53 e-05)$.

\section{Supplementary Files}

This is a list of supplementary files associated with this preprint. Click to download.

- SupplementaryTable1JASPARPROMOTERPANEL.xIs

- Listofabbreviations.docx

- SupplementaryTable2JASPARPROMOTERPANEL.xIs

- SupplementaryTable3JASPARPROMOTERPANELBAT.xIs

- SupplementaryFigure1.pdf 